\title{
Dietary factors, genetic and epigenetic influences in colorectal cancer (Review)
}

\author{
M.L. PELLEGRINI ${ }^{1}$, P. ARGIBAY ${ }^{1}$ and D.E. GOMEZ ${ }^{2}$ \\ ${ }^{1}$ Instituto de Ciencias Básicas y Medicina Experimental, Hospital Italiano de Buenos Aires; \\ ${ }^{2}$ Laboratorio de Oncología Molecular, Departamento de Ciencia y Tecnología, \\ Universidad Nacional de Quilmes, Buenos Aires, Argentina
}

Received August 17, 2009; Accepted November 10, 2009

DOI: 10.3892/etm_00000038

\begin{abstract}
Genetic influences, together with epigenetic components and dietary factors, play a fundamental role in the initiation and progression of cancer by causing a number of deregulations. Colorectal cancer (CRC) is a disease influenced by dietary factors, for which established genetic and epigenetic alterations have been identified. Within CRC, there are hereditary syndromes that present mutations in the germline hMLH1, and also alterations in the methylation of the promoters. Epigenetics has also been established as a pathway of carcinogenesis. In the present review, we analyzed studies conducted to discern the different pathways leading to established CRC, stressing the importance of identifying factors that may predict CRC at an early stage, since it is mostly a silent disease observed at the clinical level in advanced stages.
\end{abstract}

\section{Contents}

1. Introduction

2. Epigenetics

3. Genomic imprinting

4. IGF2, loss of imprinting and colon cancer

5. IGF2, loss of imprinting and microsatellite instability

6. p57 $^{\mathrm{Kip} 2}$ (CDKN1C) and colorectal cancer

7. hMLH1 epimutations

8. Folate metabolism and colorectal cancer

9. Conclusions

\section{Introduction}

Colorectal cancer (CRC) is a disease that involves genetic and epigenetic changes. CRC arises when the epithelial cells

Correspondence to: Dr Daniel E. Gomez, Laboratorio de Oncología Molecular, Departamento de Ciencia y Tecnología Universidad Nacional de Quilmes, R.S. Peña 352, Bernal (1876), Buenos Aires, Argentina

E-mail: degomez@unq.edu.ar

Key words: colorectal cancer, epigenetics, genomic imprinting of the colon or rectum begin to behave abnormally with an excessive proliferation generally resulting in the formation of an exofitic mass, mainly polyps. A polyp is defined as an abnormal grape-like formation within the inner wall of the colon or rectum. Initially these polyps are benign, but they have the potential to become malignant. Polyps grow slowly over many (3-15) years (1).

Most people do not develop polyps until they are over 50 years of age. Approximately 1 in 20 polyps can become cancerous if not removed. Therefore, prevention can be achieved by detecting the presence of polyps. CRC is one of the most common malignant tumors in humans, and has been observed to occur mainly in two specific patterns: sporadic and hereditary.

Sporadic cases account for $75-80 \%$ of the total and are the result of the accumulation of somatic mutations in oncogenes, tumor-suppressor genes and DNA-repair genes. These mutations are likely the result of dietary and environmental factors as well as aging. They tend to develop in individuals 50 years of age or older with no previous family history of the disease.

Hereditary cases account for approximately $10 \%$ of the total and include familiar adenomatous polyposis (FAP) and hereditary non-polyposis syndrome (HNPCC, or Lynch syndrome).

FAP is characterized by hundreds of polyps that present from an early age. This syndrome has an autosomal dominant character since it is caused by a dominant mutation in the APC gene (2).

The APC gene encodes a cytoplasmatic protein that regulates $\beta$-catenin. $\beta$-catenin acts primarily as a transcription activator. Under normal conditions, when the colonic epithelium remains intact and cell proliferation does not occur, most of the $\beta$-catenin forms a protein complex. The APC gene induces the phosphorylation and degradation of $\beta$-catenin not bound to the complex, generating a decrease in protein concentrations in the cell. When the APC gene is lost, it results in the accumulation of free cytoplasmic $\beta$-catenin followed by its translocation to the nucleus, activating several genes involved in cell proliferation. Therefore, the APC gene acts as a tumor-suppressor.

HNPCC is characterized by mutations in specific genes that comprise the machinery of DNA repair (hMSH2, hMSH6, hMLH1, hPMS1 and hPMS2). This is a complex enzymatic machinery that corrects errors during DNA replication. When 
one of the genes is mutated, the repair machinery does not work and results in microsatellite instability (MSI), which is a classic characteristic of this condition. Families with a member presenting HNPCC should not only undergo genetic studies to determine if they have the mutation, but should also be aware of other types of cancer that are associated with the syndrome, such as endometrial and ovarian cancer. Individuals with this mutation have a high risk of developing cancer, but do not have the same number of polyps as patients with FAP; however, the few polyps that do occur are highly likely to become malignant. In addition to the machinery of mismatch repair (MMR) gene mutations, abnormal methylation along the promoters causing gene silencing has been noted (3).

Sporadic cases show such methylation, and hereditary cases can be explained in part by Kundson's theory, which states that a second hit to a normal allele produces a change in phenotype. The first hit is inflicted by a mutation in the germline and the second hit by methylation in the promoters (4).

In recent years, epigenetic changes in addition to mutations have been proposed as possible causes of cancer. Cancer does not have a unique origin; on the contrary, many cellular changes occur that together result in the loss of normal behavior. It is therefore important to study the epigenetics of cancer to learn more about this complex disease.

Epigenetics involves changes at the genomic and chromatin levels that do not affect the sequence of nucleotides. At the genomic level, methylation occurs at specific sites, called $\mathrm{CpG}$ islands, and results in changes in gene expression. At the chromatin level, epigenetic changes occur through acetylation and deacetylation modifying gene activity (5). A few years ago, it was established that deregulations in the patterns of DNA methylation are a common feature of the neoplastic cell, producing a decrease (hypomethylation) (6) or increase (hypermethylation) (7) in the normal methylation state. These changes result in the activation of oncogenes or the inactivation of tumor-suppressor genes, leading to an imbalance in the cell metabolism. Regarding epigenetics, we also found genes that preferentially express one allele (either paternal or maternal), a feature called genomic imprinting. The differential expression of one allele is the result of methylations at the promoter regions or other regulatory areas. Changes at the chromatin level are also important; they produce a repression or relaxation of heterochromatin, thus regulating transcription. In addition to deregulation in the DNA methylation pattern, variations in the expression of imprinted genes have been found. The problem is accentuated when these genes are part of the proliferation and cell death pathways, altering those processes.

One of the characteristics of epigenetics is that it is influenced by environmental conditions. Factors that can alter the epigenome may appear early in life (in a single occurence) when they are being established, or the alterations may present in a chronic form causing later changes during adulthood. A reduction in certain components of dietary intake may also affect the epigenome. A decrease in folate concentration, the main substrate for the synthesis of amino and nucleic acids, is capable of causing a change in the pattern of DNA methylation.

Genetic mechanisms are therefore not the only pathway leading to cancer. Epigenetic changes - changes in the heritable expression pattern mediated by mechanisms that do not alter the nucleotide sequence of DNA - are being increasingly considered as an alternative to mutations and chromosomal alterations in blocking gene function.

We will now describe in detail concepts and recent advances in colorectal cancer epigenetics.

\section{Epigenetics}

Up until 1950, the term epigenetics was used to refer to all the events comprised in the development of a fertilized zygote to the constitution of a mature organism. The current definition is 'changes heritable and reversible that occur in the DNA resulting in differential expression of alleles of a gene' (8).

Changes that occur at the DNA level include methylation in specific areas of the genome and alterations in chromatin.

In bacteria, methylation is associated with the identification of particular strains, and is also used to differentiate DNA that has been replicated. In eukaryotes, several functions, including transcription regulation, $\mathrm{X}$ chromosome inactivation, maintenance of chromosome structure and silencing of parasitic DNA, are carried out.

Among cytosines, 2-7\% are methylated in mammalian DNA (9). There are areas where the density of the dinucleotide CGs is low. These regions, representing $80 \%$ of $\mathrm{CpG}$ sites, are normally methylated and are located in exons, noncoding regions and in areas of repetitive DNA. Methylation of these areas serves to silence the transcription of non-coding regions, which prevents the expression of repetitive elements of DNA and parasitic DNA, which are a threat to the integrity of the genome (7-10).

As previously mentioned, DNA methylation occurs in CG dinucleotide rich areas, called $\mathrm{CpG}$ islands, which represent $1 \%$ of the genome. These are located around promoters and non-transcribed regions. Generally, $\mathrm{CpG}$ islands are not methylated in normal cells, with the exception of the $\mathrm{X}$ chromosome and alleles silenced by genomic imprinting. These regions are frequently associated with transcriptional active areas that are not methylated (11).

DNA methylation is catalyzed by methyltransferases which add a methyl group to a cytosine preceded by a guanine. There are two types of methyltransferases: de novo methylases and maintenance methylases (Dnmt1, Dnmt2, Dnmt3a and Dnmt3b). De novo methylases place a methyl group into a new position and must recognize some specific sequence. The maintenance methylases act constitutively on hemimethylated sites, becoming fully methylated.

Changes occurring on histones that alter genetic expression are acetylations, deacetylations, methylations and phosphorylations. The enzymes involved in expression are histone acetyl transferases (HAT) and those involved in silencing are DNA methyl transferases (Dnmt) and deacetylases (HDAC).

Acetylation occurs on the lysine residues of histones. The acetyl group has a negative charge, so when it associates with lysine its positive charge is neutralized; therefore, histones bind to DNA with less force, and are more accessible to the enzymatic machinery. Deacetylation results in the opposite effect; the union between DNA and histones becomes stronger.

Histone methylation is performed by histone methyltransferase enzymes (HMTs) that methylate in arginine and lysine residues. Unlike acetylation, methylation increases the affinity of basic residues by the DNA. Methylation at lysine 9 
of histone $\mathrm{H} 3$ is associated with silent DNA and is globally distributed in heterochromatic regions, such as centromeres and telomeres.

Deacetylation is related to methylation, since HDAC is recruited by Dnmt1, 2 and 3. Moreover, in some cases, HDAC activity requires the recognition of previously methylated $\mathrm{CpG}$ sites. Since the pattern of acetylation is maintained during mitosis, it is thought that such patterns represent a heritable epigenetic imprinting that may affect genetic transcription.

This finding was confirmed by inhibiting deacetylation with the drug trichostatin (TSA), which resulted in expression of the allele that is normally an imprint of IGF2 in both mouse and human cells. In addition, there was a decrease in DNA methylation in treated cells, indicating that acetylation and DNA methylation are linked in the regulation of the process of imprinting (12).

Histone phosphorylation occurs during cell division and is present in the four histones. In certain phases of the cell cycle, specific phosphorylated histones are found to favor the condensation of chromatin. In addition, phosphorylation is involved in transcription, where histone $\mathrm{H} 3$ is phosphorylated, establishing a transcriptional competence in the early response of certain genes, such as FOS or JUN.

A small fraction of acetylated $\mathrm{H} 3$ histones are phosphorylated, suggesting that this modification contributes to the activation of genes by the stimulation of HAT activity in the same histone. Therefore, simultaneous phosphorylation and acetylation of $\mathrm{H} 3$ at the Fos and Jun loci leads to the activation of transcription. It is generally found to be associated with an increase in transcriptional activity.

The amounts of different residues that can be modified in the histones and the combination of these modifications have led to the concept of the histone code (13). The histone code, through the diversity of changes in the amino acids of histones, provides additional information regarding gene expression, creating binding sites for certain proteins that lead to an active or inactive chromatin conformation, depending on the case. For example, methylation at lysine 4 and lysine 14 as well as phosphorylation of serine 10 in histone $\mathrm{H} 3$ has been associated with gene activation, while the methylation of lysine 9 in $\mathrm{H} 3$ has been associated with gene silencing (14).

\section{Genomic imprinting}

Genomic imprinting is defined as the specific silencing of a parental allele. This silencing is caused by specific methylations on certain areas of the gene, resulting in a mark in the germline that is transmitted to offspring (15). Currently, there are approximately 30 genes known to have genomic imprinting (12).

Efstratiadis (16) initially confirmed that a normal mammalian gene exhibits this characteristic by demonstrating that a mouse with an inherited defective paternal IGF2 allele presented stunting; when the defective allele was maternal, the mouse showed normal growth - assuming that the maternal allele was not involved in the normal growth of the mouse, only the paternal allele.

Genomic imprinting is established during the development of germ cells. After fertilization, during embryo growth, differences in the methylation of alleles are maintained. In an early stage in the development of germ cells, an 'erasure' of methylation occurs. This process is followed by a restoration of methylation at a later stage $(17,18)$.

The expression of genes that have genomic imprinting is constant unless a genetic or epigenetic change occurs. When this occurs, there is an imbalance in monoallelic expression, resulting in biallelic expression or silencing. This state is known as loss of imprinting (LOI).

Many genes present genomic imprinting and are involved in various pathologies, including cancer. Deregulation of imprinting has been observed in various genes and in various types of cancers. Below is a detailed discussion of the literature regarding the most important genes in which LOI has been observed in colon cancer.

\section{IGF2, loss of imprinting and colon cancer}

Insulin-like growth factors (IGFs) are involved in the regulation of cell proliferation. This group is integrated by two factors, IGF1 and IGF2, two surface receptors (IGFIR and IGF2R) and six high-affinity proteins (IGFBP1-6). The growth factor IGF2 acts in an autocrine and paracrine manner and plays an important role in tumor tissues due to its mitogenic and antiapoptotic functions mediated by $\operatorname{IGFR}(19,20)$.

IGF2 is located on the short arm of chromosome 11p15.5. The gene has nine exons and the mature peptide consists of exons 7, 8 and 9 (21). IGF2 is transcribed by four different promoters (P1-4), resulting in several proteins of various molecular weights. Promoters P2, 3 and 4 contain $\mathrm{CpG}$ islands, and their transcription is subject to imprinting. While the P1 promoter is mainly utilized in the adult liver, it does not present genomic imprinting, resulting in both alleles being active $(22,23)$. It has been observed that the promoters P2-4 undergo methylation with aging (24).

Normal methylation was initially observed in the maternal allele; however, when samples from older individuals were analyzed, methylation was noted on the promoters of the paternal allele. This does not occur in the P1 promoter, which remains active. Since human cancer presents mainly in older populations, it may be caused by methylation spreading from one allele to another. However, this should be used as a targeted agedependent marker, since the P1 promoter remains unchanged.

The genomic imprinting of IGF2 has been well studied, since it was the first locus found to have this characteristic. It was first identified in mice, and then characterized in humans $(25,26)$. Since it is a locus involved in cell proliferation, several studies have been carried out to elucidate its possible role in the process of carcinogenesis. The focus of the present review is on studies conducted in CRC.

Early studies suggesting that genomic imprinting is a potential mechanism of disease were conducted in patients with the Beckwith-Wiedemann (27) and Prader Willi (28) syndromes. Later, it was confirmed that the LOI of IGF2 contributes to the onset and progression of these syndromes (29). The BeckwithWiedemann syndrome showed an inclination to generate embryonic tumors, such as Wilms' tumor, which is in turn characterized by loss of heterozygosity $(\mathrm{LOH})$ of chromosome 11p15.5. LOH involves the loss of genetic material; sometimes these deletions involve the loss of an entire gene and its flanking regions. 
When one of the alleles is already deleterious, heterozygosis disappears, resulting in LOH. A total of $69 \%$ of Wilms' tumors not showing LOH on chromosome 11p showed biallelic expression of IGF2, suggesting that LOI is a new epigenetic mechanism in the carcinogenesis process (30).

One of the first studies analyzing the status of IGF2 imprinting in CRC revealed that $39 \%$ of cases were informative. Among these, 38\% were found to have LOI, analyzing a polymorphism in DNA and RNA (22). Other studies have corroborated these results, finding 33\% of IGF2 LOI in CRC. These results were confirmed by immunohistochemistry when samples were compared to normal tissue (31).

The deregulation of the genomic imprinting of IGF2 is therefore involved in CRC. LOI was found with high frequency in the normal mucosa of patients with CRC, indicating that it is an alteration that occurs early in carcinogenesis (32). In the same study, IGF2 LOI in the peripheral blood of 4 patients with IGF2 LOI in tumor and normal mucosa was found.

In our studies, we also found LOI in $6 \%$ of informative peripheral blood samples. Furthermore, LOI was observed in 36 and $60 \%$ of tumor and mucosa cases, respectively. Three cases showed biallelic expression in mucosa and tumors, suggesting that the mucosa was in a period of transition towards a malignant phenotype, and another three showed LOI in tumor tissue but not in normal mucosa, suggesting that they were in a less advanced period (unpublished data).

These results indicate that, besides being a change occurring in the initiation of cancer, it can also be a change at the germline level. This suggests that the imprinting of IGF2 is a predictive marker of CRC $(33,34)$.

It has been established that IGF2 LOI occurs in CRC early and frequently in tumors of proximal location (35). No association with environmental changes has been found, suggesting that IGF2 LOI is not an environmental alteration, but rather a hereditary risk factor (36). One recent study assessed the temporal stability of the state of genomic imprinting (37). Although the sample analyzed was small, most individuals were found to have a similar state of genomic imprinting during the reported period, and no significant association was found with age. This suggests that IGF2 LOI is not an acquired phenomenon, but rather a hereditary or congenital one.

The location of the IGF2 gene is unusual, since it is located in a region adjacent to the promoter of the H19 gene. The H19 gene has a monoallelic maternal expression and, with IGF2, was one of the first genes in which genomic imprinting was identified (38). Based on comparative studies of murine and human sequences, the gene does not have an open reading frame. This allows us to conclude that the gene product is an untranslated RNA with an as yet undefined role (39).

It has been shown that a regulatory region is involved in the expression of both genes, which defines the state of genomic imprinting. This zone is called the imprinting control region (ICR) and is divided into areas that are prone to methylation, called differentially methylated regions (DMRs) (19-21). The mouse ICR is divided in four regions, while the human ICR is divided into seven DMRs (22). When DMRs are not methylated, a factor called CTCF binds to the ICR.

The CTCF factor acts as a transcriptional insulator factor which sets boundaries between the enhancer and promoter of specific genes (40). Once it binds to DNA, the CTCF factor act as a repressor or an activator depending on the binding site. When the DMRs of the maternal allele are unmethylated, the CTCF binds and isolates the enhancers of IGF2, resulting in the expression of H19. The opposite occurs in the paternal allele, where expression of IGF2 is exhibited. In this way, the imprinting of both loci is regulated. This has been called 'competition between enhancers'.

The IGF2 gene moreover presents DMRs itself (DMR0, 1 and 2), regulating its expression. DMR0, one of the most studied DMRs, is located upstream of a complex of promoters that are under the control of imprinting. This region is homologous to a mouse region on the maternal allele that has been observed to be methylated (dmr0) (41). Initially, studies in humans found the same pattern of methylation and identified loss of methylation of the DMR0 accompanied by IGF2 LOI, suggesting that there are other areas that regulate gene expression in addition to regulation from the ICR.

Deregulation of genomic imprinting involves the biallelic expression of the three promoters with genomic imprinting. It was noted that these three were unsuppressed simultaneously in the maternal allele (42). Since the difference in methylation is upstream of the first promoter imprinted, it has been suggested that this area is a center that acts in cis, causing silencing of the allele (43).

Corroborating these results, DMR0 hypomethylation has been found in osteosarcome (44), breast (45) and ovarian (46) cancer, but without an association with IGF2 LOI, suggesting that the mechanism for deregulating expression is independent of LOI. Cui et al also found IGF2 LOI to be more highly associated with the hypomethylation of DMR0 of IGF2 than the methylation of H19, suggesting that IGF2 DMR is the most important for the maintenance of imprinting in CRC (47). These results imply that the regulatory mechanisms of imprinting are likely to be independent of the enhancer competition model. Possibly, genomic imprinting in this region is regulated by DMRs in IGF2 rather than by those located between both genes.

However, methylation has been found in DMR0 on the paternal allele $(48,49)$. Methylation status in this area has been postulated as a possible predictive indicator of cancer, since a higher prevalence of DMR0 hypomethylation than of LOI was found, and since $36 \%$ of CRC cases presented hypomethylation of DMR0 with monoallelic expression (50).

Deregulation of genomic imprinting is a well-established feature not only of human cancer, but also of other pathologies. In CRC, IGF2 LOI constitutes the first step in carcinogenesis, causing uncontrolled cell proliferation. Changes in methylation may be caused by mutations in proteins that maintain the pattern of methylation, or by external factors.

Inactivation of genomic imprinting can occur under conditions of cellular stress. Cell proliferation under stress can lead to permanent genetic and epigenetic changes. It has been shown that in primary cultures exposed to stress, the expression profile of all genes was stable except for the genes that presented genomic imprinting (51).

\section{IGF2, loss of imprinting and microsatellite instability}

Microsatellites are tandem simple sequences that appear throughout the human genome. The repeats involve 2-6 
dinucleotides (52). Microsatellites play roles in chromatin organization, the regulation of DNA metabolic processes and gene regulation (53).

Replication machinery is prone to malfunction in regions containing microsatellites, causing mutations when these alterations are not recognized and repaired by the machinery of mismatch repair (MMR). In such cases, additions or deletions of the microsatellite repeats occur, resulting in a change in the size of the microsatellite.

This change is called microsatellite instability (MSI). MSI is a situation in which a microsatellite allele in the germline wins or loses repeat units and changes its somatic length. MSI is one of the pathways in which the genome is destabilized, and this destabilization has been suggested to be an early prerequisite for carcinogenesis (54).

MSI is an indicator of the typical clonal expansion observed in cancer, since the alteration can be detected only when many cells are affected by the same change. MSI is produced by the loss of function of MMR genes, mainly hMLH1 or hMSH2.

The loss of function of MMR proteins results in the widespread accumulation of somatic mutations throughout the genome. These mutations sometimes occur in oncogenes and tumor-suppressor genes that play a key role in the initiation and progression of cancer $(55,56)$. In Lynch syndrome, the MSI phenotype requires the double biallelic inactivation of the MMR genes. The first inactivation involves a mutation in the germline. The second inactivation is due to three possible mechanisms: $\mathrm{LOH}$, somatic mutations or epigenetic alterations. Changes at the epigenomic level occur through the methylation of the hMLH1 promoter, and lead to its silencing (57). MSI in sporadic CRC is present in $15 \%$ of cases and occurs through the methylation of the promoter of both alleles of hMLH1 (7).

In 1997, the United States Cancer Institute established a panel of five microsatellite markers (Bat 26, Bat 25, D2S123, D5S346 and D17S250) to determine the stage of MSI in tumors $(58,59)$. When instability is found in two or more microsatellites it is called MSI-H, when it is observed in one microsatellite it is called MSI-L, and when there is no observed instability it is termed microsatellite stable (MSS). Analysis of Bat-26 or Bat-25 is sufficient for detecting most MSI-H cases (60).

It has been established in several studies that tumors presenting MSI-H have a higher prevalence in the proximal colon and present a rapid alteration from benign polyps to malignant ones; however, these tumors are associated with increased patient survival as compared with MSS or MSI-L tumors $(61,62)$. It has been suggested that this more favorable prognosis is due to the fact that MSI-H tumors are more sensitive to chemotherapy.

MSI is therefore directly related to epigenetic alterations, since methylation of the gene promoter of MMR genes is an established feature of CRC. It is possible that, in addition to the occurrence of aberrant methylation in the MMR genes, abnormal methylation also occurs at the sites of regulation of imprinting. In such cases, we propose that LOI occurs before MSI. Otherwise, the MSI state occurs first, with the subsequent disruption of the regulation of imprinting. Significant associations have been demonstrated between LOI and MSI. Nishihara et al (63) found that a high frequency of cases presenting MSI also presented IGF2 LOI as compared with MSS cases.

Cui et al (32) found IGF2 LOI in $91 \%$ of MSI cases and in $12 \%$ of MSS cases. Since MSI is present in $16-37 \%$ of sporadic cases while somatic mutations occur in only $16 \%$, they proposed that other genetic or epigenetic factors affect different loci, promoting a state of instability. For example, alterations in genes that encode chromatin factors can affect DNA replication, and hence the fidelity of the imprinting causing MSI and LOI.

Nakagawa et al (64) confirmed previous results and noted that the majority of sporadic MSI(+) CRC with IGF2 LOI showed hypermethylation of the hMLH1 and p16 promoters. A minority of MSI(-) CRC cases exhibited IGF2 LOI (4/37, $11 \%$ ) and methylation of $\mathrm{p} 16$, indicating that $\mathrm{p} 16$ methylation is strongly associated with LOI even in MSI(-) cases. Thus, the $\mathrm{CpG}$ island methylator phenotype (CIMP) affects not only p16 and hMLH1, but also the imprinting control region, resulting in LOI.

However, Ohta et al (35) found that several CRC cases with IGF2 MSI were located in the distal colon, while CRC cases with IGF2 LOI were located in the proximal colon (22.7 vs. $56.6 \%$ ), corroborating previous findings. However, they did not find a significant association between MSI and IGF2 LOI, possibly because they analyzed a very small number of MSI(+) CRC cases.

Finally, Sasaki et al (65) found IGF2 LOI with a significant predominance in the right side and in poorly differentiated carcinomas. Since MSI is present in cases in the same location, it would be expected that cases with LOI also showed MSI. However, they did not find any association, and so suggested that IGF2 LOI is involved in the development of CRC but must belong to a different pathway.

Therefore, more research is needed to determine whether an association exists between LOI and MSI. Although IGF2 is not part of the group of genes which have the characteristic CIMP(+), this methylated locus is found with biallelic expression. This methylation must be produced through a different pathway, rather than the already described CIMP.

\section{6. p57 Kip2 (CDKN1C) and colorectal cancer}

The cell cycle is regulated by a series of proteins known as cyclins, cyclin-dependent kinases (CDK) and cyclindependent kinase inhibitors (CDKI). Together, these proteins coordinate the sequence of transitions of the cell cycle.

The activity of kinases depends upon their union with the appropriate cyclin; therefore, kinases act as positive regulators of cyclin activity. CDKIs act as negative regulators and play an important role in cell cycle progression.

CDKIs are grouped according to their structure and their various targets. There are two classes, the INK4 class (p16 ${ }^{\mathrm{INK} 4 \mathrm{a}}$, $\mathrm{p} 15^{\mathrm{INK} 4 \mathrm{~b}}, \mathrm{p} 18^{\mathrm{INK} 4 \mathrm{c}}$ and $\mathrm{p} 19^{\text {INK4d }}$ ), which inhibits only the catalytic subunits of CDK4 and CDK6, and the second class, Cip/ Kip, which is capable of inhibiting cell cycle progression by binding and inhibiting CDK complexes in the G1 phase, the cyclins D, E and A. The Cip/Kip family is composed of the following inhibitors: p21 ${ }^{\mathrm{Cip} 1}, \mathrm{p} 27^{\mathrm{Kip} 1}$ and $\mathrm{p} 57^{\mathrm{Kip} 2}$ (66). Due to the role of $\mathrm{p} 57^{\mathrm{Kip} 2}$, its eventual loss contributes to accelerated cell proliferation. 
The $\mathrm{p} 57^{\mathrm{Kip} 2}$ protein is localized in the nucleus and has been found to have tissue-specific expression, unlike p21 $1^{\mathrm{Cip} 1}$ and p $27^{\text {Kipl }}$ (67). The locus is located on chromosome $11 \mathrm{p} 15.5$, and there have been no reports of any somatic mutations except normal genetic variations (68).

Significant differences in the expression of $\mathrm{p} 57^{\mathrm{Kip} 2}$ compared with normal tissue have been found. Since no mutations were found in this gene, it was suggested that the low expression was likely due to post-transcriptional and posttransductional modifications. It was also proposed that the gene had genomic imprinting (69).

The genomic imprinting of $\mathrm{p} 57^{\mathrm{Kip} 2}$ was first confirmed in mice, where it was found that the paternal allele was methylated and repressed. In mice, the gene is located in the distal region of chromosome 7 , in a cluster of genes including IGF2, H19 and Mash2 that show genomic imprinting (70).

The same locus has been characterized in humans. Genomic imprinting was also found to be present, expressing the maternal allele preferentially, but it was noted that the imprinting was not absolute; the paternal allele is expressed at low levels in some tissues and at comparable levels with the maternal allele in fetal brain tissue and in some embryonic tissues (71).

Of the CDKIs, $\mathrm{p} 57^{\mathrm{Kip} 2}$ is the only one to show genomic imprinting (72). After confirming that the p57 $7^{\text {Kip2 }}$ gene is a genomic imprinted locus located in an area frequently disrupted in cancer, studies involving various malignancies were conducted, some of them focused on CRC (73).

In Wilms' tumors, there was an absence of this protein in all the samples analyzed as compared with normal tissue (74). In hepatic cancer, a significant decrease in the expression of p57 $7^{\mathrm{Kip} 2}$ was also found. This decrease was associated with high biological aggressiveness (75).

In breast and stomach cancer, mutations were not observed, but the levels of mRNA were significantly decreased (76). These results suggest that epigenetic alterations rather than mutations are more important in the inactivation of p57 Kip2.

LOI of $\mathrm{p} 57^{\mathrm{Kip} 2}$ was observed in head and neck cancer in $13 \%$ of cases, while monoallelic expression was observed in the normal mucosa associated with each tumor (77).

In CRC, few studies have been carried out. A significant correlation was found between the low expression of $\mathrm{p} 57^{\mathrm{Kip} 2}$ and tumor size (78). In addition, it has been shown that the protein expression of $\mathrm{p} 57^{\mathrm{Kip} 2}$ is increased in samples from normal mucosa compared to adenomas, but decreases when it moves from adenoma to primary carcinoma samples. This suggests that the loss of p57 $7^{\mathrm{Ki} 2}$ occurs late in colorectal carcinogenesis (79). These results have also been observed in thyroid and ovarian cancer $(80,81)$.

All mechanisms involved in the inactivation observed for the loss of protein expression involved changes in the methylation of the genome. In gastric cell lines treated with HDAC inhibitors (TSA or n-butyric), the activation of mRNA expression was noted and, upon treatment with demethylating agents (5-aza-2'-deoxycytidine), an increase in expression was observed. These results suggest that $\mathrm{p} 57^{\mathrm{Kip} 2}$ is inactivated by the formation of heterochromatin with HDAC, and that the methylation of the promoter may also be involved (82). In addition, $\mathrm{CpG}$ island methylation was observed in a region of initiation of transcription in CRC cell lines; when these were treated with 5 azacitidine, expression was restored. In the same study, it was determined by immunoprecipitation with anti-AC $\mathrm{H} 3$ and $\mathrm{H} 4$ that histone deacetylation was present in the methylated promoter region, corroborating the hypothesis that genomic methylation and histone deacetylation play an important role in $\mathrm{p} 57^{\mathrm{Kip} 2}$ silencing (55).

\section{7. hMLH1 epimutations}

Epimutations are defined as hemiallelic methylations that occur in the germline of a gene. Mutations of the hMLH1 gene are involved in hereditary non-polyposis syndrome (HNPCC). In patients with HNPCC, either both alleles are mutated or only one. In the latter case, the remaining normal allele may be subjected to a second inactivation produced by another point mutation or by an epigenetic alteration at the promoter level.

The term epimutation was first coined in a study in which the authors found a case with hMLH1 hypermethylation in peripheral blood among a pool of 14 cases suspected to have HNPCC. This case was MSI(+) and did not have any mutations in the MMR genes (83). When the authors examined the tumor tissue of this case, they found hypermethylation in one allele, while the other had a methylated promoter but showed LOH. Since they did not have samples from the patient's relatives, they were unable to determine the inheritance of the methylated allele, but demonstrated that methylation occurred in other tissues that were not neoplastic.

Vertical transmission was demonstrated when an epimutation was found in the sperm of a patient with multiple tumors, all with weaknesses in the MMR system but without mutations at that level (84).

In one study, hemiallelic methylation was found in peripheral blood, in hair follicles and in oral mucosa, and was assumed to be a somatic alteration. Although the methylated allele found was maternal, when mothers and other relatives were studied, the same variation was not found, suggesting that the epimutation appeared de novo (85).

Hitchins et al also demonstrated vertical transmission in a family with a mother who presented the epimutation in all somatic cells (86). Two of the sons had the epimutation with a maternal origin. This had reverted, resulting in the biallelic expression of hMLH1. In the third son, who had also inherited the epimutation from his mother, there was no reversal, leaving him at high risk of losing monoallelic expression. Researchers studied his sperm without finding the epimutation at that level. Apparently, the normal process of gametogenesis allows the correction of the epimutation by erasing the methylation of the imprinted genes in the primordial germ cells. This is observed in sons who do not show the hemimethylation, while having inherited the maternal allele. Therefore, it reflects a resistance to reprogramming through an incomplete erase or retention of an epigenetic memory. Epimutations appear to be reversible between generations, presenting a non-Mendelian inheritance.

\section{Folate metabolism and colorectal cancer}

Epigenetics, through the methylation of DNA, is part of the initiation and progression of CRC. The folate metabolism is 
the main pathway for obtaining the substrate for DNA methylation, and is restricted mostly to components of the diet. Migration studies have shown that people who migrate from countries with low risk of CRC to countries with higher risk adopt the CRC risk rate of the country to which they emigrated within one or two generations. In these studies, the main focus was alterations in dietary intake, though environmental conditions may also confer different activities to the same genes. Therefore, we can infer a relationship between nutritional and epigenetic factors $(87,88)$.

The monocarbon reserve of the cell is very important, since it depends on the synthesis of amino acids, purines, pyrimidines and the generation of methionine. All of these molecules are vital for most metabolic pathways, and their production depends entirely on what the cell obtains from the external environment.

In order to transform the carbon unit into molecules, it must be activated by a carrier. Therefore, the monocarbon reserve includes carbon units attached to carriers. The major carriers are carbon folate and S-adenosylmethyonine (SAM) (89).

SAM is a high energy compound formed by the condensation of methionine with ATP. SAM represents the principal methyl donor to DNA, RNA, hormones and neurotransmitters. However, the active form of folate is 5,6,7,8 tetrahydrofolate (THF).

Folate and methionine must be obtained from the diet. Therefore, a balanced diet is important to incorporate these compounds for a correct metabolic function. The richest sources of folate are leafy vegetables, fruit, yeast and liver (90).

Based on our observation of methylation variations (hypoand hypermethylations) in cancer, it is extremely important to study the folate metabolism in CRC. It has been hypothesized that alterations in the methylation of the genome can be produced, in some cases, by a decrease in these nutrients in the diet $(53,91,92)$.

Although the results of several studies are contradictory, it is essential to ascertain whether there is an association between folate metabolism and cancer, as environmental factors in primary tumors are central to sporadic carcinogenesis.

The first step in folate metabolism is the reduction of folate to THF through dihidrofolate reductase. Then, one unit of carbon from serine or glycine is transferred to THF to form 5,10-methylenetetrahydrofolate.

5,10-Methylenetetrahydrofolate is used for the synthesis of thymidine and purines, or is reduced to 5-methyl-THF by the enzyme methylenetetrahydrofolate reductase (MTHFR), which is used to methylate the homocysteine to form methionine via methionine synthase. It then forms SAM and is used for DNA methylation.

The folate metabolism can be disrupted by various factors, such as a reduction in substrates, mutations in the enzymes involved in the process, or a decrease in the enzyme activities due to polymorphisms.

It is known that certain gene polymorphisms produce a reduction in enzyme activity that is encoded. For example, the MTHFR has a germline variant which includes a substitution of a cytosine for a thymidine at position 677 , converting an alanine to valine in the protein sequence. This change results in a thermolabile enzyme with a decrease in their activity (93).
It has been reported that individuals homozygous for the TT variant have an enzyme activity of $30 \%$, while that of CT heterozygote individuals is $65 \%$ (94). A reduction in activity leads to a decrease in the substrate used for the methylation of the homocysteine, thus generating SAM. Theoretically, a reduction in the substrate for the methylation of DNA would be observed, hence hypomethylation of the genome would be seen; an effect observed in CRC.

There are several studies which have confirmed an association between a reduced risk of CRC and the MTHFR TT genotype, suggesting a protective effect of the $\mathrm{T}$ allele $(86,89,95,96)$. In a study conducted with individuals with Lynch syndrome who had a confirmed mutation of the MMR gene and were homozygous for the wild-type allele of MTHFR, CRC developed earlier than in individuals with one or two copies of the $\mathrm{T}$ allele, corroborating results regarding its protective effect (97). However, contrary finding were found in studies where the TT genotype was determined to be a risk factor (98).

Methylated phenotype (CIMP), a feature of CRC, involves the hypermethylation of specific $\mathrm{CpG}$ islands of specific promoters, leading to the silencing of gene transcription. Abnormal methylation of the hMLH1 gene promoter is an example of CIMP, resulting in the majority of sporadic CRC cases with a MSI(+) phenotype. It has been observed in various studies that individuals with the MTHFR 677TT genotype are more susceptible to developing CRC by the microsatellite instability pathway $(99,100)$. This association is probably due to an alteration in DNA methylation resulting in the abnormal methylation of specific promoters, such as hMLH1. Confirming these results, promoter hypermethylation of hMLH1 in patients with the 677TT genotype was observed $(101,102)$.

Although a strong association was found between the TT genotype and MSI(+) cases, such a relationship was independent of the methylation status of hMLH1 (103). Furthermore, it was demonstrated that the relationship between MSI and $677 \mathrm{TT}$ is influenced by the intake of folate.

Among individuals with an adequate intake of folate, combination of the two MTHFR polymorphisms (C677T and A1298C) has been associated with a decreased risk of MSI(+) CRC. This protective effect was not observed in individuals with low folate intake (104).

The current hypothesis asserts that when there is a good intake of folate, although the activity of MTHFR is low, enough folate is converted to 5-methylenetetrahydrofolate to cover the needs of methyl groups in metabolism. However, an association was not found between the MTHFR polymorphism and MSI status. It was proposed that the reduced activity of MTHFR favors the synthesis of DNA and the MMR systems, reducing the erroneous incorporation of uracil and resulting in stable (MSS) tumors.

The methionine synthase (MS) gene also presents polymorphisms (85). This gene has a polymorphism (A919G) at position 2756 , resulting in a change of asparagine to glycine at position 919 of the transcript (105).

The amino acid sequence is therefore altered in a functional site, causing the functional alteration of the enzyme (106). MS is part of the folate metabolic pathway, methylating the homocysteine to methionine using a methyl group donated by 5-methylenetetrahydrofolate. 
The A919G polymorphism has been associated with an increase in homocysteine levels. It has been noted that individuals with cancer who have the GG genotype have a significant reduction in the methylation of $\mathrm{CpG}$ islands in tumor-suppressor genes (107).

There are no direct associations between homozygous genotypes for the polymorphism and the risk of CRC $(108,109)$. However, when a polymorphism in MS reductase was analyzed, a correlation was observed.

The MS gene may be inactive due to the oxidation of its cofactor, vitamin B12. In order to function again, when oxidation has occurred, the gene depends on the remethylation of vitamin B12 via methionine synthase reductase (MSR) (110). It has been observed that allelic variants of the MSR gene (A66G) generate an enzyme with reduced affinity for MS, associated with a decreased risk of CRC (111).

Therefore, the activity of the MS gene may be altered not only by a polymorphism within the gene, but also by a polymorphism in an enzyme that regulates the activity of the protein. In another study, researchers found no association between the A919G polymorphism and the risk of CRC, but did detect a decreased risk of CRC in the presence of polymorphisms that resulted in a decrease in the activity of MTHFR with low expression of thymidine synthase (TS). The decrease in enzyme TS expression involved a decrease in DNA synthesis, and thus a reduction in cell replication (112).

It can therefore be concluded that, when the deregulation of the folate metabolism is one of the causes of abnormal methylation observed in neoplastic cells, this abnormal methylation is not only due to one polymorphism in the enzymes involved in the pathway, but may rather be caused by an interaction between several gene polymorphisms or the imbalance of several enzyme activities involved in the process.

\section{Conclusions}

Epigenetics is increasingly considered a cause of cancer since it involves changes that do not alter the sequence of the DNA and that are potentially reversible. It is vital to identify alterations which precede the onset of cancer, thus allowing us to eliminate the suffering related to cancer.

The first step towards achieving this goal was the discovery of the loss of IGF2 imprinting in normal tissue adjacent to tumors, suggesting that this change occurs at the onset of carcinogenesis. Next, loss of IGF2 imprinting in peripheral blood was demonstrated, indicative of change at the germline level. Loss of imprinting may therefore act as a biological marker for the early detection of cancer.

The second step should be to clarify the mechanisms by which epigenetic patterns are established, to determine which areas are actually involved in the process of silencing of an allele. In the case of IGF2, it was initially thought that the regulation only occurred in the area shared with H19. However, it has now been established that there are other areas in this gene that are differentially methylated, that regulate its expression independent of the theory of competition between enhancers.

The reversal of methylations through the use of certain drugs is a potential solution in need of further study. Researchers are likely capable of changing the disturbance that occurs and results in pathology, but can also produce the deregulation of other areas causing other problems. Thus, initially, epigenetics may provide us with answers related to the diagnosis and prognosis of cancer patients, even if it cannot yet be applied in the treatment of cancer.

Dietary factors are also related to epigenetics, and are perhaps the most important aspects of the regulation system. Changes in diet affect the pool of substrates for methylation. It has been shown that a good folate intake prevents CRC.

It is very important to continue studies along these lines, since the discovery of a predictive feature using a simple blood test would enormously facilitate patient diagnosis. Understanding the properties of tumors, such as imprinting status, microsatellite instability and the presence of epimutations, would provide information regarding the prognosis of the disease as well as tailored treatments according to the epigenetic status of the tumor. In the future, epigenetic alterations observed in pathologies will provide us with a new framework within which to work for the benefit of the patient.

\section{Acknowledgements}

Dr D.E. Gomez is a member of CONICET. The present work was partially financed by the Fundacion para el Desarrollo de las Ciencias Básicas (FUCIBA), a non-profit organization.

\section{References}

1. Lynch HT and de la Chapelle A: Hereditary colorectal cancer. N Engl J Med 348: 919-932, 2003.

2. Calvert PM and Frucht H: The genetics of colorectal cancer. Ann Intern Med 137: 603-612, 2002.

3. Lynch HT, Lanspa S, Smyrk T, Boman B, Watson P and Lynch J: Hereditary nonpolyposis colorectal cancer (Lynch syndromes I \& II). Genetics, pathology, natural history and cancer control, Part I. Cancer Genet Cytogenet 53: 143-160, 1991.

4. Terdiman JP, Conrad PG and Sleisenger MH: Genetic testing in hereditary colorectal cancer: indications and procedures. Am J Gastroenterol 94: 2344-2356, 1999.

5. Cheung P and Lau P: Epigenetic regulation by histone methylation and histone variants. Mol Endocrinol 19: 563-573, 2005.

6. Hoffmann MJ and Schulz WA: Causes and consequences of DNA hypomethylation in human cancer. Biochem Cell Biol 83: 296-321, 2005.

7. Herman JG and Baylin SB: Gene silencing in cancer in association with promoter hypermethylation. N Engl J Med 349: 2042-2054, 2003.

8. Holliday R: Epigenetics: a historical overview. Epigenetics 1: 76-80, 2006.

9. Esteller M: Relevance of DNA methylation in the management of cancer. Lancet Oncol 4: 351-358, 2003.

10. Robertson KD and Wolffe AP: DNA methylation in health and disease. Nat Rev Genet 1: 11-19, 2000.

11. Warnecke PM and Bestor TH: Cytosine methylation and human cancer. Curr Opin Oncol 12: 68-73, 2000.

12. Hoffman AR, Vu TH and Hu J: Mechanisms of genomic imprinting. Growth Horm IGF Res 10: S18-S19, 2000

13. Jenuwein T and Allis CD: Translating the histone code. Science 293: 1074-1080, 2001

14. Kondo Y and Issa JP: Epigenetic changes in colorectal cancer. Cancer Metastasis Rev 23: 29-39, 2004.

15. Sasaki H, Allen ND and Surani MA: DNA methylation and genomic imprinting in mammals. EXS 64: 469-486, 1993.

16. Efstratiadis A: Parental imprinting of autosomal mammalian genes. Curr Opin Genet Dev 4: 265-280, 1994.

17. Reik W and Walter J: Genomic imprinting: parental influence on the genome. Nat Rev Genet 2: 21-32, 2001.

18. Constancia M, Pickard B, Kelsey G and Reik W: Imprinting mechanisms. Genome Res 8: 881-900, 1998.

19. Pollak M: Insulin-like growth factor physiology and neoplasia. Growth Horm IGF Res 10: S6-S7, 2000.

20. Pavelic K, Bukovic D and Pavelic J: The role of insulin-like growth factor 2 and its receptors in human tumors. Mol Med 8: 771-780, 2002. 
21. Van Dijk MA, van Schaik FM, Bootsma HJ, Holthuizen P and Sussenbach JS: Initial characterization of the four promoters of the human insulin-like growth factor II gene. Mol Cell Endocrinol 81: 81-94, 1991.

22. Kinouchi Y, Hiwatashi N, Higashioka S, Nagashima F, Chida M and Toyota T: Relaxation of imprinting of the insulin-like growth factor II gene in colorectal cancer. Cancer Lett 107: 105-108, 1996.

23. Sussenbach JS, Steenbergh PH, Jansen E, et al: Structural and regulatory aspects of the human genes encoding IGF-I and -II Adv Exp Med Biol 293: 1-14, 1991.

24. Issa JP, Vertino PM, Boehm CD, Newsham IF and Baylin SB Switch from monoallelic to biallelic human IGF2 promoter methylation during aging and carcinogenesis. Proc Natl Acad Sci USA 93: 11757-11762, 1996.

25. DeChiara TM, Robertson EJ and Efstratiadis A: Parental imprinting of the mouse insulin-like growth factor II gene. Cell 64: 849-859, 1991.

26. Giannoukakis N, Deal C, Paquette J, Goodyer CG and Polychronakos C: Parental genomic imprinting of the human IGF2 gene. Nat Genet 4: 98-101, 1993.

27. Henry I, Bonaiti-Pellie C, Chehensse V, et al: Uniparental paternal disomy in a genetic cancer-predisposing syndrome. Nature 351: 665-667, 1991.

28. Nicholls RD, Knoll JH, Butler MG, Karam S and Lalande M: Genetic imprinting suggested by maternal heterodisomy in nondeletion Prader-Willi syndrome. Nature 342: 281-285, 1989.

29. Cerrato F, Sparago A, Verde G, et al: Different mechanisms cause imprinting defects at the IGF2/H19 locus in BeckwithWiedemann syndrome and Wilms' tumour. Hum Mol Genet 17: 1427-1435, 2008.

30. Rainier S, Johnson LA, Dobry CJ, Ping AJ, Grundy PE and Feinberg AP: Relaxation of imprinted genes in human cancer. Nature 362: 747-749, 1993

31. Takano Y, Shiota G and Kawasaki H: Analysis of genomic imprinting of insulin-like growth factor 2 in colorectal cancer Oncology 59: 210-216, 2000

32. Cui H, Horon IL, Ohlsson R, Hamilton SR and Feinberg AP Loss of imprinting in normal tissue of colorectal cancer patients with microsatellite instability. Nat Med 4: 1276-1280, 1998.

33. Kawakami K, Ruszkiewicz A, Bennett G, et al: DNA hypermethylation in the normal colonic mucosa of patients with colorectal cancer. Br J Cancer 94: 593-598, 2006.

34. Cui H, Cruz-Correa M, Giardiello FM, et al: Loss of IGF2 imprinting: a potential marker of colorectal cancer risk. Science 299: 1753-1755, 2003.

35. Ohta M, Sugimoto T, Seto M, et al: Genetic alterations in colorectal cancers with demethylation of insulin-like growth factor II. Hum Pathol 39: 1301-1308, 2008.

36. Cruz-Correa M, Cui H, Giardiello FM, et al: Loss of imprinting of insulin growth factor II gene: a potential heritable biomarker for colon neoplasia predisposition. Gastroenterology 126: 964-970, 2004

37. Cruz-Correa M, Zhao R, Oviedo M, et al: Temporal stability and age-related prevalence of loss of imprinting of the insulin-like growth factor-2 gene. Epigenetics 4: 114-118, 2009.

38. Bartolomei MS, Zemel S and Tilghman SM: Parental imprinting of the mouse H19 gene. Nature 351: 153-155, 1991.

39. Brannan CI, Dees EC, Ingram RS and Tilghman SM: The product of the H19 gene may function as an RNA. Mol Cell Biol 10: 28-36, 1990

40. Kuhn EJ and Geyer PK: Genomic insulators: connecting properties to mechanism. Curr Opin Cell Biol 15: 259-265, 2003.

41. Moore T, Constancia M, Zubair M, et al: Multiple imprinted sense and antisense transcripts, differential methylation and tandem repeats in a putative imprinting control region upstream of mouse Igf2. Proc Natl Acad Sci USA 94: 12509-12514, 1997.

42. Taniguchi T, Schofield AE, Scarlett JL, Morison IM, Sullivan MJ and Reeve AE: Altered specificity of IGF2 promoter imprinting during fetal development and onset of Wilms tumour. Oncogene 11: 751-756, 1995

43. Sullivan MJ, Taniguchi T, Jhee A, Kerr $N$ and Reeve AE: Relaxation of IGF2 imprinting in Wilms tumours associated with specific changes in IGF2 methylation. Oncogene 18: 7527-7534, 1999.

44. Ulaner GA, Vu TH, Li T, et al: Loss of imprinting of IGF2 and H19 in osteosarcoma is accompanied by reciprocal methylation changes of a CTCF-binding site. Hum Mol Genet 12: 535-549, 2003.

45. Byun HM, Wong HL, Birnstein EA, Wolff EM, Liang G and Yang AS: Examination of IGF2 and H19 loss of imprinting in bladder cancer. Cancer Res 67: 10753-10758, 2007.
46. Murphy SK, Huang Z, Wen Y, et al: Frequent IGF2/H19 domain epigenetic alterations and elevated IGF2 expression in epithelial ovarian cancer. Mol Cancer Res 4: 283-292, 2006.

47. Cui $\mathrm{H}$, Onyango $\mathrm{P}$, Brandenburg $\mathrm{S}$, Wu Y, Hsieh $\mathrm{CL}$ and Feinberg AP: Loss of imprinting in colorectal cancer linked to hypomethylation of H19 and IGF2. Cancer Res 62: 6442-6446, 2002.

48. Monk D, Sanches R, Arnaud P, et al: Imprinting of IGF2 P0 transcript and novel alternatively spliced INS-IGF2 isoforms show differences between mouse and human. Hum Mol Genet 15: 1259-1269, 2006.

49. Murrell A, Ito Y, Verde G, et al: Distinct methylation changes at the IGF2-H19 locus in congenital growth disorders and cancer. PLoS One 3: e1849, 2008.

50. Ito $\mathrm{Y}$, Koessler $\mathrm{T}$, Ibrahim $\mathrm{AE}$, et al: Somatically acquired hypomethylation of IGF2 in breast and colorectal cancer. Hum Mol Genet 17: 2633-2643, 2008.

51. Pantoja C, de Los Rios L, Matheu A, Antequera F and Serrano M: Inactivation of imprinted genes induced by cellular stress and tumorigenesis. Cancer Res 65: 26-33, 2005.

52. De la Chapelle A: Microsatellite instability. N Engl J Med 349: 209-210, 2003.

53. Ehrlich M: DNA methylation in cancer: too much, but also too little. Oncogene 21: 5400-5413, 2002.

54. Alonso A, Moreno S, Valiente A, Artigas M, Perez-Juana A and Ramos Arroyo MA: Genetic mechanisms in the hereditary predisposition to colorectal cancer. An Sist Sanit Navar 29: 59-76, 2006.

55. Kikuchi T, Toyota M, Itoh F, et al: Inactivation of p57KIP2 by regional promoter hypermethylation and histone deacetylation in human tumors. Oncogene 21: 2741-2749, 2002.

56. Perucho M: Tumors with microsatellite instability: many mutations, targets and paradoxes. Oncogene 22: 2223-2225, 2003.

57. Kaz A, Kim YH, Dzieciatkowski S, et al: Evidence for the role of aberrant DNA methylation in the pathogenesis of Lynch syndrome adenomas. Int J Cancer 120: 1922-1929, 2007.

58. Boland CR, Thibodeau SN, Hamilton SR, et al: A National Cancer Institute Workshop on Microsatellite Instability for cancer detection and familial predisposition: development of international criteria for the determination of microsatellite instability in colorectal cancer. Cancer Res 58: 5248-5257, 1998.

59. Perucho M: Correspondence ref: Boland CR, et al: A National Cancer Institute workshop on microsatellite instability for cancer detection and familial predisposition: development of international criteria for the determination of microsatellite instability in colorectal cancer. Cancer Res 58: 5248-5257, 1998. Cancer Res 59: 249-256, 1999.

60. Hoang JM, Cottu PH, Thuille B, Salmon RJ, Thomas G and Hamelin R: BAT-26, an indicator of the replication error phenotype in colorectal cancers and cell lines. Cancer Res 57: 300-303, 1997.

61. Ribic CM, Sargent DJ, Moore MJ, et al: Tumor microsatelliteinstability status as a predictor of benefit from fluorouracil-based adjuvant chemotherapy for colon cancer. N Engl J Med 349: 247-257, 2003.

62. Lothe RA, Peltomaki P, Meling GI, et al: Genomic instability in colorectal cancer: relationship to clinicopathological variables and family history. Cancer Res 53: 5849-5852, 1993.

63. Nishihara S, Hayashida T, Mitsuya K, et al: Multipoint imprinting analysis in sporadic colorectal cancers with and without microsatellite instability. Int J Oncol 17: 317-322, 2000.

64. Nakagawa H, Chadwick RB, Peltomaki P, Plass C, Nakamura Y and de La Chapelle A: Loss of imprinting of the insulin-like growth factor II gene occurs by biallelic methylation in a core region of H19-associated CTCF-binding sites in colorectal cancer. Proc Natl Acad Sci USA 98: 591-596, 2001.

65. Sasaki J, Konishi F, Kawamura YJ, Kai T, Takata O and Tsukamoto T: Clinicopathological characteristics of colorectal cancers with loss of imprinting of insulin-like growth factor 2 . Int J Cancer 119: 80-83, 2006.

66. Sherr CJ and Roberts JM: CDK inhibitors: positive and negative regulators of G1-phase progression. Genes Dev 13: 1501-1512, 1999.

67. Lee MH, Reynisdottir I and Massague J: Cloning of p57KIP2, a cyclin-dependent kinase inhibitor with unique domain structure and tissue distribution. Genes Dev 9: 639-649, 1995.

68. Reid LH, Crider-Miller SJ, West A, Lee MH, Massague J and Weissman BE: Genomic organization of the human p57KIP2 gene and its analysis in the G401 Wilms' tumor assay. Cancer Res 56: 1214-1218, 1996. 
69. Orlow I, Iavarone A, Crider-Miller SJ, et al: Cyclin-dependent kinase inhibitor p57KIP2 in soft tissue sarcomas and Wilms' tumors. Cancer Res 56: 1219-1221, 1996.

70. Hatada I and Mukai T: Genomic imprinting of p57KIP2, a cyclindependent kinase inhibitor, in mouse. Nat Genet 11: 204-206, 1995.

71. Hatada I, Inazawa J, Abe T, et al: Genomic imprinting of human p57KIP2 and its reduced expression in Wilms' tumors. Hum Mol Genet 5: 783-788, 1996.

72. Cost GJ, Thompson JS, Reichard BA, Lee JY and Feinberg AP: Lack of imprinting of three human cyclin-dependent kinase inhibitor genes. Cancer Res 57: 926-929, 1997.

73. Matsuoka S, Thompson JS, Edwards MC, et al: Imprinting of the gene encoding a human cyclin-dependent kinase inhibitor, p57KIP2, on chromosome 11p15. Proc Natl Acad Sci USA 93: 3026-3030, 1996.

74. Thompson JS, Reese KJ, DeBaun MR, Perlman EJ and Feinberg AP: Reduced expression of the cyclin-dependent kinase inhibitor gene p57KIP2 in Wilms' tumor. Cancer Res 56 5723-5727, 1996.

75. Ito $\mathrm{Y}$, Takeda T, Sakon M, Tsujimoto M, Monden $\mathrm{M}$ and Matsuura N: Expression of p57/Kip2 protein in hepatocellular carcinoma. Oncology 61: 221-225, 2001.

76. Shin JY, Kim HS, Lee KS, et al: Mutation and expression of the $\mathrm{p} 27 \mathrm{KIP} 1$ and p57KIP2 genes in human gastric cancer. Exp Mol Med 32: 79-83, 2000.

77. Lai S, Goepfert H, Gillenwater AM, Luna MA and El-Naggar AK: Loss of imprinting and genetic alterations of the cyclin-dependent kinase inhibitor p57KIP2 gene in head and neck squamous cell carcinoma. Clin Cancer Res 6: 3172-3176, 2000.

78. Noura S, Yamamoto H, Sekimoto M, et al: Expression of second class of KIP protein p57KIP2 in human colorectal carcinoma. Int J Oncol 19: 39-47, 2001.

79. Li JQ, Wu F, Usuki H, et al: Loss of p57KIP2 is associated with colorectal carcinogenesis. Int J Oncol 23: 1537-1543, 2003.

80. Ito $\mathrm{Y}$, Yoshida H, Nakano K, et al: Expression of p57/Kip2 protein in normal and neoplastic thyroid tissues. Int $\mathrm{J}$ Mol Med 9: 373-376, 2002.

81. Sui L, Dong Y, Ohno M, Watanabe Y, Sugimoto K and Tokuda M: Expression of p57kip2 and its clinical relevance in epithelial ovarian tumors. Anticancer Res 22: 3191-3196, 2002.

82. Shin JY, Kim HS, Park J, Park JB and Lee JY: Mechanism for inactivation of the KIP family cyclin-dependent kinase inhibitor genes in gastric cancer cells. Cancer Res 60: 262-265, 2000.

83. Gazzoli I, Loda M, Garber J, Syngal S and Kolodner RD: A hereditary nonpolyposis colorectal carcinoma case associated with hypermethylation of the MLH1 gene in normal tissue and loss of heterozygosity of the unmethylated allele in the resulting microsatellite instability-high tumor. Cancer Res 62: 3925-3928, 2002.

84. Suter CM, Martin DI and Ward RL: Germline epimutation of MLH1 in individuals with multiple cancers. Nat Genet 36: 497-501, 2004

85. Hitchins M, Williams R, Cheong K, et al: MLH1 germline epimutations as a factor in hereditary nonpolyposis colorectal cancer. Gastroenterology 129: 1392-1399, 2005.

86. Hitchins MP, Wong JJ, Suthers G, et al: Inheritance of a cancerassociated MLH1 germ-line epimutation. N Engl J Med 356: 697-705, 2007

87. Kolonel LN: Cancer patterns of four ethnic groups in Hawaii. J Natl Cancer Inst 65: 1127-1139, 1980.

88. Kmet J: The role of migrant population in studies of selected cancer sites: a review. J Chronic Dis 23: 305-324, 1970.

89. Slattery ML, Potter JD, Samowitz W, Schaffer D and Leppert M: Methylenetetrahydrofolate reductase, diet and risk of colon cancer. Cancer Epidemiol Biomarkers Prev 8: 513-518, 1999.

90. Hubner RA and Houlston RS: Folate and colorectal cancer prevention. Br J Cancer 100: 233-239, 2009.

91. Feinberg AP, Gehrke CW, Kuo KC and Ehrlich M: Reduced genomic 5-methylcytosine content in human colonic neoplasia. Cancer Res 48: 1159-1161, 1988.

92. Giovannucci E: Epidemiologic studies of folate and colorectal neoplasia: a review. J Nutr 132: S2350-S2355, 2002.

93. Chen J, Giovannucci E, Kelsey K, et al: A methylenetetrahydrofolate reductase polymorphism and the risk of colorectal cancer. Cancer Res 56: 4862-4864, 1996.
94. Frosst P, Blom HJ, Milos R, et al: A candidate genetic risk factor for vascular disease: a common mutation in methylenetetrahydrofolate reductase. Nat Genet 10: 111-113, 1995.

95. Ma J, Stampfer MJ, Giovannucci E, et al: Methylenetetrahydrofolate reductase polymorphism, dietary interactions and risk of colorectal cancer. Cancer Res 57: 1098-1102, 1997.

96. Chen J, Giovannucci E, Hankinson SE, et al: A prospective study of methylenetetrahydrofolate reductase and methionine synthase gene polymorphisms, and risk of colorectal adenoma. Carcinogenesis 19: 2129-2132, 1998.

97. Pande M, Chen J, Amos CI, Lynch PM, Broaddus R and Frazier ML: Influence of methylenetetrahydrofolate reductase gene polymorphisms C677T and A1298C on age-associated risk for colorectal cancer in a caucasian lynch syndrome population. Cancer Epidemiol Biomarkers Prev 16: 1753-1759, 2007.

98. Osian G, Procopciuc L and Vlad L: MTHFR polymorphisms as prognostic factors in sporadic colorectal cancer. J Gastrointestin Liver Dis 16: 251-256, 2007.

99. Shannon B, Gnanasampanthan S, Beilby J and Iacopetta B: A polymorphism in the methylenetetrahydrofolate reductase gene predisposes to colorectal cancers with microsatellite instability. Gut 50: 520-524, 2002.

100. Hubner RA, Lubbe S, Chandler I and Houlston RS: MTHFR C677T has differential influence on risk of MSI and MSS colorectal cancer. Hum Mol Genet 16: 1072-1077, 2007.

101. Oyama K, Kawakami K, Maeda K, Ishiguro K and Watanabe G: The association between methylenetetrahydrofolate reductase polymorphism and promoter methylation in proximal colon cancer. Anticancer Res 24: 649-654, 2004.

102. Kawakami K, Ruszkiewicz A, Bennett G, Moore J, Watanabe G and Iacopetta B: The folate pool in colorectal cancers is associated with DNA hypermethylation and with a polymorphism in methylenetetrahydrofolate reductase. Clin Cancer Res 9: 5860-5865, 2003

103. Clarizia AD, Bastos-Rodrigues L, Pena HB, et al: Relationship of the methylenetetrahydrofolate reductase C677T polymorphism with microsatellite instability and promoter hypermethylation in sporadic colorectal cancer. Genet Mol Res 5: 315-322, 2006.

104. Eaton AM, Sandler R, Carethers JM, Millikan RC, Galanko J and Keku TO: 5,10-Methylenetetrahydrofolate reductase 677 and 1298 polymorphisms, folate intake, and microsatellite instability in colon cancer. Cancer Epidemiol Biomarkers Prev 14: 2023-2029, 2005

105. Li YN, Gulati S, Baker PJ, Brody LC, Banerjee R and Kruger WD: Cloning, mapping and RNA analysis of the human methionine synthase gene. Hum Mol Genet 5: 1851-1858, 1996.

106. Chen LH, Liu ML, Hwang HY, Chen LS, Korenberg J and Shane B: Human methionine synthase. cDNA cloning, gene localization and expression. J Biol Chem 272: 3628-3634, 1997.

107. Ma J, Stampfer MJ, Christensen B, et al: A polymorphism of the methionine synthase gene: association with plasma folate, vitamin B12, homocyst(e)ine and colorectal cancer risk. Cancer Epidemiol Biomarkers Prev 8: 825-829, 1999.

108. Paz MF, Avila S, Fraga MF, et al: Germ-line variants in methylgroup metabolism genes and susceptibility to DNA methylation in normal tissues and human primary tumors. Cancer Res 62: 4519-4524, 2002.

109. Matsuo K, Hamajima N, Hirai T, et al: Methionine synthase reductase gene A66G polymorphism is associated with risk of colorectal cancer. Asian Pac J Cancer Prev 3: 353-359, 2002.

110. Leclerc D, Wilson A, Dumas R, et al: Cloning and mapping of a cDNA for methionine synthase reductase, a flavoprotein defective in patients with homocystinuria. Proc Natl Acad Sci USA 95: 3059-3064, 1998.

111. Olteanu H, Munson T and Banerjee R: Differences in the efficiency of reductive activation of methionine synthase and exogenous electron acceptors between the common polymorphic variants of human methionine synthase reductase. Biochemistry 41: 13378-13385, 2002.

112. Ulrich CM, Curtin K, Potter JD, Bigler J, Caan B and Slattery ML: Polymorphisms in the reduced folate carrier, thymidylate synthase, or methionine synthase and risk of colon cancer. Cancer Epidemiol Biomarkers Prev 14: 2509-2516, 2005 . 